\title{
Rayleigh-Taylor instability of classical diffusive density profiles for miscible fluids in porous media: a linear stability analysis
}

\author{
P. M. J. Trevelyan(i) - A. De Wit(i) - J. Kent
}

Received: 25 June 2020 / Accepted: 3 September 2021

(C) The Author(s), under exclusive licence to Springer Nature B.V. 2021

\begin{abstract}
A Rayleigh-Taylor instability typically develops when a denser layer overlies a less dense one in the gravity field. In that case, the initial base state density profile is a step function for which linear stability analysis results are well known. We investigate here analytically the linear stability analysis of other classical diffusive density profiles for porous media flows. We find that, for a species A initially distributed in the upper half of the domain with an initial concentration profile of $(-X)^{m}$ for $0<m<1$ where $X$ is the vertical coordinate, and absent from the bottom half of the domain, for large times the eigenfunctions grow like $\exp \left(\omega_{0} T^{(m+1) / 2}+\omega_{1} \ln (T)\right)$ where $\omega_{0}$ and $\omega_{1}$ are constants and $T$ is time. Thus, the growth rate defined by $(1 / A)(\mathrm{d} A / \mathrm{d} T)$ decays like $c_{1} T^{(m-1) / 2}+c_{2} T^{(m-2) / 3}$ whilst the maximum growing wavenumber scales with $T^{(m-2) / 6}$. These results are compared to the growth rates obtained using numerical linear stability analysis. Our analytical predictions provide a set of generalised results that pave the way to the analysis of Rayleigh-Taylor instabilities of nontrivial density profiles.
\end{abstract}

Keywords Buoyancy $\cdot$ Darcy's law $\cdot$ Linear stability $\cdot$ Porous media $\cdot$ Self similar

PACS 47.20.Bp $\cdot 47.56 .+\mathrm{r} \cdot 52.35 . \mathrm{Py}$

\section{Introduction}

A Rayleigh-Taylor (RT) instability can occur whenever a denser fluid overlies a less dense miscible fluid in a gravity field. The resulting instability deforms a flat interface into fingers [1-4]. RT instabilities are present in many situations including inside the Earth's mantle [5], the oceans [6], and the atmosphere [7], they can be on smaller scales in reactive porous media fluids [8] or on larger scales within stars [9].

P. M. J. Trevelyan $(\bowtie) \cdot$ J. Kent

Division of Mathematics \& Statistics, University of South Wales, Pontypridd, Wales CF37 1DL, UK

e-mail: Philip.Trevelyan@southwales.ac.uk

Present address:

J. Kent

Met Office, Exeter, UK

A. De Wit

Nonlinear Physical Chemistry Unit, Service de Chimie Physique et Biologie Théorique, Faculté des Sciences, Université Libre de Bruxelles (ULB), CP 231, 1050 Brussels, Belgium 
The onset condition for an RT instability in a finite domain in the presence of linear density profiles was obtained in $[10,11]$. When the initial vertical density profile between two infinite homogeneous layers in a porous medium is a step function, the dispersion relation giving the growth rate of the instability as a function of the wavenumber has been derived analytically for $t=0$ [12] and numerically at later times [13] using the quasi steady state approximation (QSSA), where the base state profiles were assumed frozen in time. In the case of Hele-Shaw cells (two glass plates separated by a thin gap), for various fluid flow models, Martin et al. [14] provided analytical dispersion relations for the particular cases of either a linear or a step function profile. The influence of the width of the diffusive mixing zone on the wavelength of the instability at the onset of instability has been discussed in [15].

The first experimental studies of the RT destabilisation of a stratification of a sugar or salt aqueous solution above water in a Hele-Shaw cell were carried out by Hill [16] and Wooding [17]. A comparison between the experimental and linear stability analysis of the RT instability in a Hele-Shaw cell starting from a step profile of a denser solution on top of a less dense one was performed by Fernandez et al. [18]. Experiments analysing the RT instability of stable linear profiles of concentration around a step profile [19] or a variety of initial density profiles [20,21] have also been performed for turbulent flows.

Recently, there has been growing interest to understand the stability of more complicated initial diffusive profiles in porous media flows. Indeed, differential diffusion [22] or chemical reactions [23,24] for instance have been shown experimentally in Hele-Shaw cells and numerically to be able to trigger rapidly non-monotonic density profiles in which extrema in the density profile develop as soon as the two layers are in contact. For buoyancydriven instabilities, [13] found that the time when the product of the maximum instantaneous growth rate and time reached unity was a useful time to compare the linear stability analysis with nonlinear simulations as the system is then still in the early stages of the instability. In the case of an initial step function profile [25], the linear stability analysis predicted, in dimensionless units that the product of the maximum instantaneous growth rate and time reached unity around time 100 , and even at time 400 , the nonlinear simulations still showed a very weak instability. It is, thus, possible to put in contact two zones and let it evolve towards the self-similar diffusion solution before a buoyancy-driven RT instability starts to grow.

Using the QSSA [26], Gandhi et al. [27] analytically obtained the onset condition for a RT instability at time $t=0$ for several different piecewise constant density profiles. Even though the QSSA has been shown to fail at small times, Kim [28] found that it shows good agreement with spectral analysis methods at larger times. In the same spirit, Cowell et al. [25] employed the QSSA to numerically examine the stability of several different evolving density profiles in a porous medium in an infinite domain. For an initial step function density profile, they numerically found that for large times, the growth rate scaled with time to the power of $-(1 / 2)$, which agreed with the work by Ben et al. [29] which used the spectral analysis method for viscous fingering. Cowell et al. [25] also numerically examined the stability of a finite layer density profile and numerically found that, for large times, the instantaneous growth rate was inversely proportional to time, which means that the system does not grow exponentially in time but instead grows algebraically in time. Biferale et al. [21] investigated the generic case of an initially unstable vertical temperature distribution with general power-law singular initial conditions, providing insight into situations when the RT mixing develops with a nonuniform background like in a thermally stratified atmosphere.

These works that have examined the RT instability for nontrivial density profiles, have all been performed either experimentally or numerically. Hence, the ability to examine the stability of simple classical diffusive profiles to obtain analytical predictions to which to compare the numerical analysis is of interest. In this context, we analytically examine the time scalings for a RT instability of various diffusive density profiles in an infinite domain. The analytical stability analysis allows one to verify the numerical analysis which has taken place previously. In this study, we consider the case when a species is initially only located in the upper half of the domain and its concentration is proportional to the vertical height to the power of $m$ where $m$ is a constant between 0 and 1 . Physically this corresponds to a nonlinear concentration profile which increases more slowly than a linear profile. Such initial profiles evolve by diffusion to self-similar profiles, as illustrated in Fig. 1.

We assume that the instability is weak so that the concentration profile is diffusing in time before the RT instability sets in. This can be obtained experimentally in a horizontal set-up (for instance in Hele-Shaw cells) flipped by a tiny angle with regard to the horizontal. 
(a)

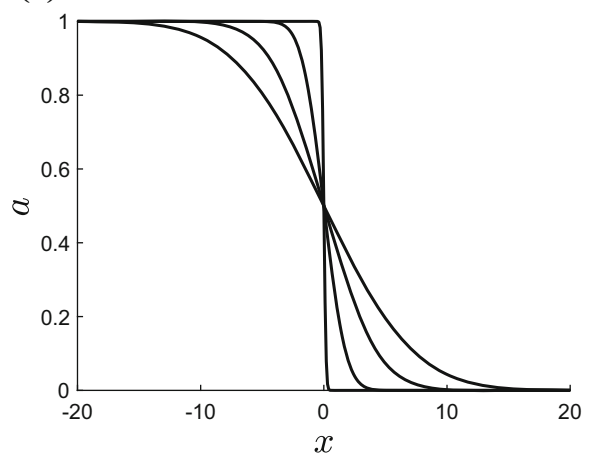

(b)

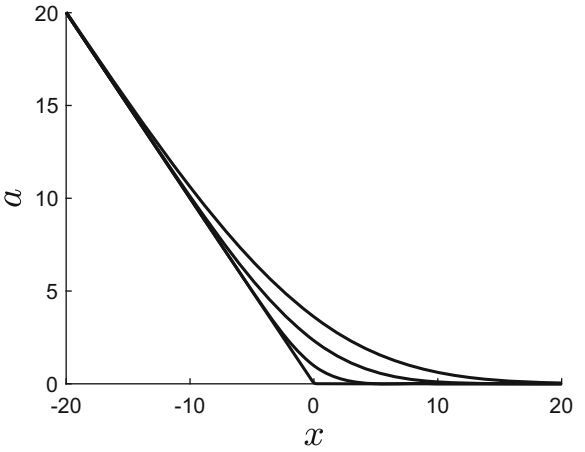

Fig. 1 Sketch of concentration profiles: a complementary error function, b a semi-linear profile. Increasing $x$ is downwards

In Sect. 2, the governing equations are given, non-dimensionalised and presented in terms of the stream function. In Sect. 3, the base state solution is presented. In Sect. 4, nonlinear simulations are included to demonstrate the time scale before the onset of the instability. In Sect. 5, a large time asymptotic linear stability analysis is developed. In Sect. 6, a numerical linear stability analysis is used to compare with the scalings predicted by the large time asymptotic linear stability analysis. Finally, we draw our conclusions in Sect. 7.

\section{Model}

Suppose that a species A is dissolved in a fluid contained inside a two-dimensional homogeneous porous medium. We assume that the permeability $K$ of the porous medium is constant. The fluid flow is assumed to satisfy Darcy's law and the concentration, $a$, of species A satisfies the mass transport equation, see [30]. For Darcy's law to be valid for flow in a porous medium, it is required that the flow length scales are larger than the typical pore size, so we require that the wavelengths of any instabilities are much larger than the pore size. We assume that the dispersion coefficient $D$ of species A is a constant.

The fluid density $\rho$ is assumed to be a linear function of the concentration of species A. The concentration of the species is assumed sufficiently small that the Boussinesq approximation is valid and so the flow can be considered incompressible, in addition to the dynamic viscosity $\mu$ being constant. The vertical coordinate $x$ is chosen to increase in the downwards direction. This problem is modelled by the following equations:

$$
\begin{aligned}
& \underline{\nabla} \cdot \underline{u}=0, \\
& \underline{\nabla} p=-\frac{\mu}{K} \underline{u}+\rho g \underline{i}, \\
& \frac{\partial a}{\partial t}+\underline{u} \cdot \underline{\nabla} a=D \nabla^{2} a, \\
& \rho=\rho_{0}(1+\alpha a)
\end{aligned}
$$

where $p$ is the fluid's pressure, $t$ is time, $g$ is the magnitude of the acceleration due to gravity, and $\underline{i}$ is the unit downwards pointing vector along the $x$ axis. Here $\rho_{0}$ is the density of the solvent and $\alpha$ is the solutal expansion coefficient defined by $\alpha=\left(1 / \rho_{0}\right)(\mathrm{d} \rho / \mathrm{d} a)$. We assume that species A increases the fluids density, i.e. $\alpha>0$.

For convenience, we assume that the domain is sufficiently large to be assumed infinite. We assume that initially the fluid is at rest and that the concentration of the species is given by

$a(x, y, 0)=a_{0}(-x)^{m} H(-x)$,

where $y$ is the horizontal coordinate, $a_{0}$ is a constant, $H$ is the Heaviside step function and $0<m<1$. Note that, for $m=0$, we recover the initial step function shown in Fig. 1a. The far-field conditions are

$$
\underline{u} \rightarrow \underline{0} \text { as } x \rightarrow \pm \infty,
$$




$$
\begin{aligned}
& a \rightarrow 0 \text { as } x \rightarrow \infty \\
& a \rightarrow a_{0}(-x)^{m} \text { as } x \rightarrow-\infty
\end{aligned}
$$

\subsection{Non-dimensionalisation}

We non-dimensionalise the system by rescaling the variables as follows:

$\underline{u}=\frac{D}{L} \underline{U}, \quad p=\rho_{0} g L X+\frac{\mu D}{K} P, \quad a=a_{0} L^{m} A$,

$x=L X, \quad y=L Y, \quad t=\frac{L^{2}}{D} T$ where $L=\left(\frac{\mu D}{\alpha \rho_{0} a_{0} g K}\right)^{1 /(m+1)}$,

where $L$ is the characteristic length scale of the problem. We introduce a stream function $\psi$ such that the velocity is given by $\underline{U}=\left(\psi_{Y},-\psi_{X}\right)$. Taking the curl of Darcy's law eliminates the pressure and the resulting system of equations is

$\nabla^{2} \psi=A_{Y}$,

$A_{T}+\psi_{Y} A_{X}-\psi_{X} A_{Y}=\nabla^{2} A$.

The dimensionless initial profile is

$A(X, Y, 0)=(-X)^{m} H(-X)$.

The far-field conditions are non-dimensionalised to become

$\psi \rightarrow 0$ as $X \rightarrow \pm \infty$,

$A \rightarrow 0$ as $X \rightarrow \infty$,

$A \rightarrow(-X)^{m}$ as $X \rightarrow-\infty$.

\section{Diffusive base state solutions}

We assume the fluid is initially at rest and the base state concentration $\tilde{A}$ is one dimensional and so satisfies the diffusion equation

$\tilde{A}_{T}=\tilde{A}_{X X}$.

We find that the base state solution is given by

$$
\tilde{A}=T^{m / 2} G^{m}(s) \text { where } s=\frac{X}{\sqrt{T}}
$$

where $G^{m}$ satisfies $2 G_{s s}^{m}+s G_{s}^{m}-m G^{m}=0$. Two linearly independent solutions of this equation are $\Omega_{1}$ and $\Omega_{2}$ given by

$\left[\Omega_{1}(s), \Omega_{2}(s)\right]=\frac{\sqrt{2} \mathrm{e}^{-s^{2} / 8}}{\sqrt{\pi s}}\left[M\left(-\frac{1+2 m}{4}, \frac{1}{4}, \frac{s^{2}}{4}\right), W\left(-\frac{1+2 m}{4}, \frac{1}{4}, \frac{s^{2}}{4}\right)\right]$,

where $M$ and $W$ are the Whittaker $\mathrm{M}(\gamma, \nu, \zeta)$ and $\mathrm{W}(\gamma, \nu, \zeta)$ functions which satisfy $4 \zeta^{2} \Psi_{\zeta \zeta}$ $-\left(\zeta^{2}-4 \gamma \zeta+4 v^{2}-1\right) \Psi=0$, as given by equation (13.1.31) in Abramowitz and Stegun [31].

The general solution is constructed using a linear combination of $\Omega_{1}$ and $\Omega_{2}$. For the function to be real and continuous at $s=0$, we choose the form:

$G^{m}(s)=c_{1} \Omega_{1}(-s) H(-s)+c_{2} \Omega_{2}(|s|)$. 
Table 1 Some values of $\lambda_{m}$ and $-G_{s}^{m}\left(\lambda_{m}\right)$ for different values of $m$

\begin{tabular}{llc}
\hline$m$ & $\lambda_{m}$ & $-G_{s}^{m}\left(\lambda_{m}\right)$ \\
\hline 0 & 0 & $(4 \pi)^{-1 / 2}=0.282095$ \\
0.25 & -0.482902 & 0.330557 \\
0.5 & -1.081804 & 0.429335 \\
0.75 & -1.929683 & 0.614720 \\
1 & $-\infty$ & 1 \\
\hline
\end{tabular}

In order for Eq. (16) to satisfy the far-field condition (14), we require that $c_{1}=2^{m+1} \Gamma(1+(m / 2))$ using Appendix A, where $\Gamma$ is the Gamma function see Eq. (6.1.1) in Abramowitz and Stegun [31], defined by $\Gamma(v)=\int_{0}^{\infty} t^{v-1} \mathrm{e}^{-t} \mathrm{~d} t$. Further, for the first derivative of $G^{m}$ to be continuous at $s=0$ we require that $c_{2}=2^{m-1} \Gamma(1+(m / 2)) \Gamma((1+m) / 2) / \sqrt{\pi}$. Thus,

$G^{m}(s)=2^{m-1} \Gamma\left(1+\frac{m}{2}\right)\left(4 H(-s) \Omega_{1}(-s)+\Gamma\left(\frac{1+m}{2}\right) \frac{\Omega_{2}(|s|)}{\sqrt{\pi}}\right)$.

Three interesting limits of Eq. (18) are

$$
\begin{aligned}
G^{0}(s)= & \frac{1}{2} \operatorname{erfc}\left(\frac{s}{2}\right), \\
G^{1}(s)= & \frac{1}{\sqrt{\pi}} \mathrm{e}^{-s^{2} / 4}-\frac{s}{2} \operatorname{erfc}\left(\frac{s}{2}\right), \\
G^{\frac{1}{2}}(s)= & \frac{|s|^{3 / 2}}{4 \sqrt{2 \pi}} \mathrm{e}^{-s^{2} / 8}\left(K\left(\frac{3}{4}, \frac{s^{2}}{8}\right)-K\left(\frac{1}{4}, \frac{s^{2}}{8}\right)\right)(2 H(s)-1) \\
& \quad+\frac{|s|^{3 / 2} \sqrt{\pi}}{4} \mathrm{e}^{-s^{2} / 8}\left(I\left(\frac{1}{4}, \frac{s^{2}}{8}\right)+I\left(-\frac{3}{4}, \frac{s^{2}}{8}\right)\right) H(-s),
\end{aligned}
$$

where $I(v, \zeta)$ and $K(v, \zeta)$ are the modified Bessel functions of the first and second kinds that satisfy $\zeta^{2} \Psi^{\prime \prime}+\zeta \Psi^{\prime}-$ $\left(\zeta^{2}+v^{2}\right) \Psi=0$.

In this study, we are going to be interested in the region where $G_{s}^{m}$ is most negative, as this corresponds to where the gradient of the concentration profile is most negative and where the system is most unstable. Hence, we define $\lambda_{m}$ to be the solution to $G_{s s}^{m}\left(\lambda_{m}\right)=0$ so that $\lambda_{m}$ is the value of $s$ at the inflection point where the gradient of $G^{m}$ is most negative. In Table 1, we include some illustrative values of $\lambda_{m}$ and $-G_{s}^{m}\left(\lambda_{m}\right)$ for different values of $m$. One notes that to a good approximation

$G_{s}^{m}\left(\lambda_{m}\right) \approx-(4 \pi)^{(1+0.7272 m)(m-1) / 2}$,

which has a maximum absolute error of about 0.0074 around $m=0.5$.

\section{Nonlinear simulations}

Simulations were obtained by numerically solving Eqs. (9) and (10). To ensure the system was perturbed, smallscale random noise of amplitude $10^{-4}$ was added to the initial concentration $A$. The stream function $\psi$ was obtained from Eq. (9) using a multi-grid method [32]. Using the Lin-Rood method [33] with a third-order finite-difference operator [34], the concentration $A$ was obtained from Eq. (10) by solving it in conservative form.

In Fig. 2, we illustrate the evolution of the instability when $m=0$ at various times $T$. At time $T=1000$, Fig. 2 shows that the instability is still very weak and essentially the system is in a purely diffusive regime. However, by time $T=2000$, the early stages of the instability are now visible, and at time $T=3000$, the system is in the 

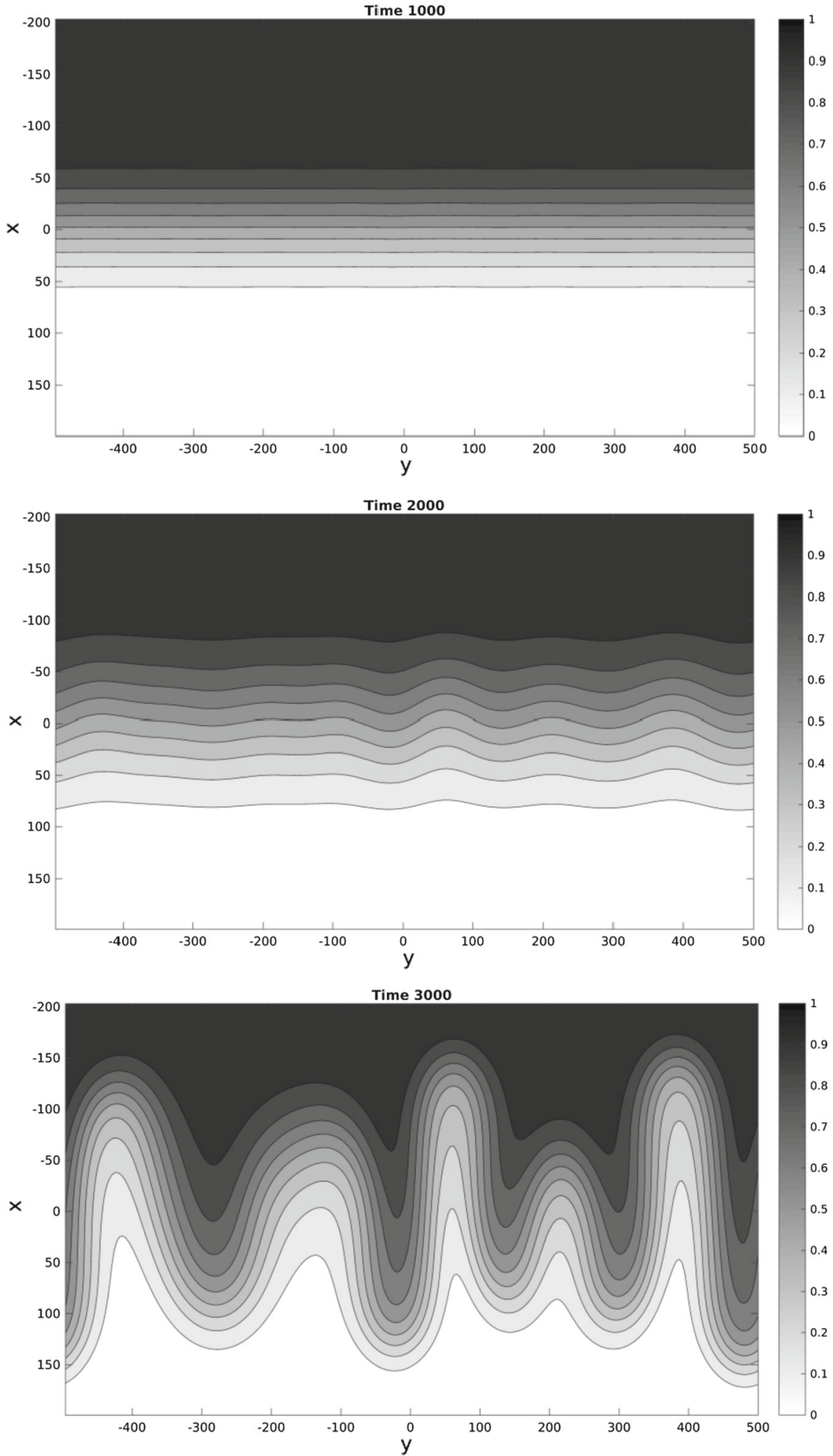

Fig. 2 Nonlinear simulations illustrating the evolution of the instability when $m=0$ in a domain $-500<y<500$ at times $T=1000$, 2000, and 3000 
very nonlinear regime. Hence, when $m=0$, one would expect a linear stability analysis to still be valid up to time $T=1000$.

In Fig. 3, we illustrate the evolution of the instability when $m=1 / 2$ at various times $T$. We see that here, the instability starts to develop already by $T=125$. At $T=100$, the profiles are barely modulated, so we expect the linear stability analysis to still be valid up to time $T=100$.

\section{Large time asymptotic linear stability}

Supposing that a diffusive base state solution exists in the system and that this stratification is put in the gravity field, we aim now to analyse analytically its stability with regard to a RT instability. To do so, we consider a small perturbation to the base state solutions of the form

$\psi=\epsilon \hat{\psi}(X, Y, T)$ and $A=\tilde{A}(X, T)+\epsilon \hat{A}(X, Y, T)$,

where $\epsilon$ is a small constant and $\tilde{A}=T^{m / 2} G^{m}(s)$. Substituting these expressions into Eqs. (9) and (10), then linearising in $\epsilon$ gives

$$
\begin{aligned}
\hat{\psi}_{X X}+\hat{\psi}_{Y Y} & =\hat{A}_{Y}, \\
\hat{A}_{T}+\tilde{A}_{X} \hat{\psi}_{Y} & =\hat{A}_{X X}+\hat{A}_{Y Y}
\end{aligned}
$$

where $\tilde{A}_{X}=T^{(m-1) / 2} G_{s}^{m}(s)$, along with the far-field boundary conditions

$\hat{A}, \hat{\psi} \rightarrow 0 \quad$ as $\quad X \rightarrow \pm \infty$.

As the instability can be very weak at small times, we are going to seek a large time asymptotic solution to allow us to approximate the stability of the system for large times, whilst still being in the early stages of the instability. Hence, we introduce the new variables

$$
\begin{aligned}
& \hat{\psi}(X, Y, T)=\tau^{q} f(\eta, \theta, \tau), \quad \hat{A}(X, Y, T)=a(\eta, \theta, \tau), \\
& X=\lambda_{m} \sqrt{\tau}+\eta \tau^{b}, \quad Y=\tau^{r} \theta \quad \text { and } \quad T=\tau,
\end{aligned}
$$

where $b, q$, and $r$ are constants to be determined. Notice we are supposing that the position of the instability is located around a moving coordinate centred around $X=\lambda_{m} \sqrt{\tau}$, i.e. where the gradient of $G^{m}(s)$ is most negative. Subbing the new variables into Eq. (19) yields

$\tau^{q-2 b} f_{\eta \eta}+\tau^{q-2 r} f_{\theta \theta}=\tau^{-r} a_{\theta}$.

In order to balance all of these terms, we require that

$q=r=b$,

then the equation becomes

$f_{\eta \eta}+f_{\theta \theta}=a_{\theta}$.

Subbing the new variables into Eq. (20) yields

$a_{\tau}-\left[\frac{\lambda_{m}}{2 \tau^{\frac{1}{2}+q}}+\frac{q \eta}{\tau}\right] a_{\eta}-\frac{q \theta}{\tau} a_{\theta}+\frac{f_{\theta}}{\tau^{(1-m) / 2}} G_{s}^{m}\left(\lambda_{m}+\frac{\eta}{\tau^{\frac{1}{2}-q}}\right)=\frac{a_{\eta \eta}+a_{\theta \theta}}{\tau^{2 q}}$.

To balance the diffusion terms on the right-hand side with the last term on the left-hand side, we require that

$q=\frac{1-m}{4}$.

Subbing in $q$ and multiplying the equation by $\tau^{(1-m) / 2}$ yields

$$
a_{\tau} \tau^{(1-m) / 2}-\left[\frac{\lambda_{m}}{2 \tau^{(1+m) / 4}}+\frac{q \eta}{\tau^{(1+m) / 2}}\right] a_{\eta}-\frac{q \theta}{\tau^{(1+m) / 2}} a_{\theta}
$$



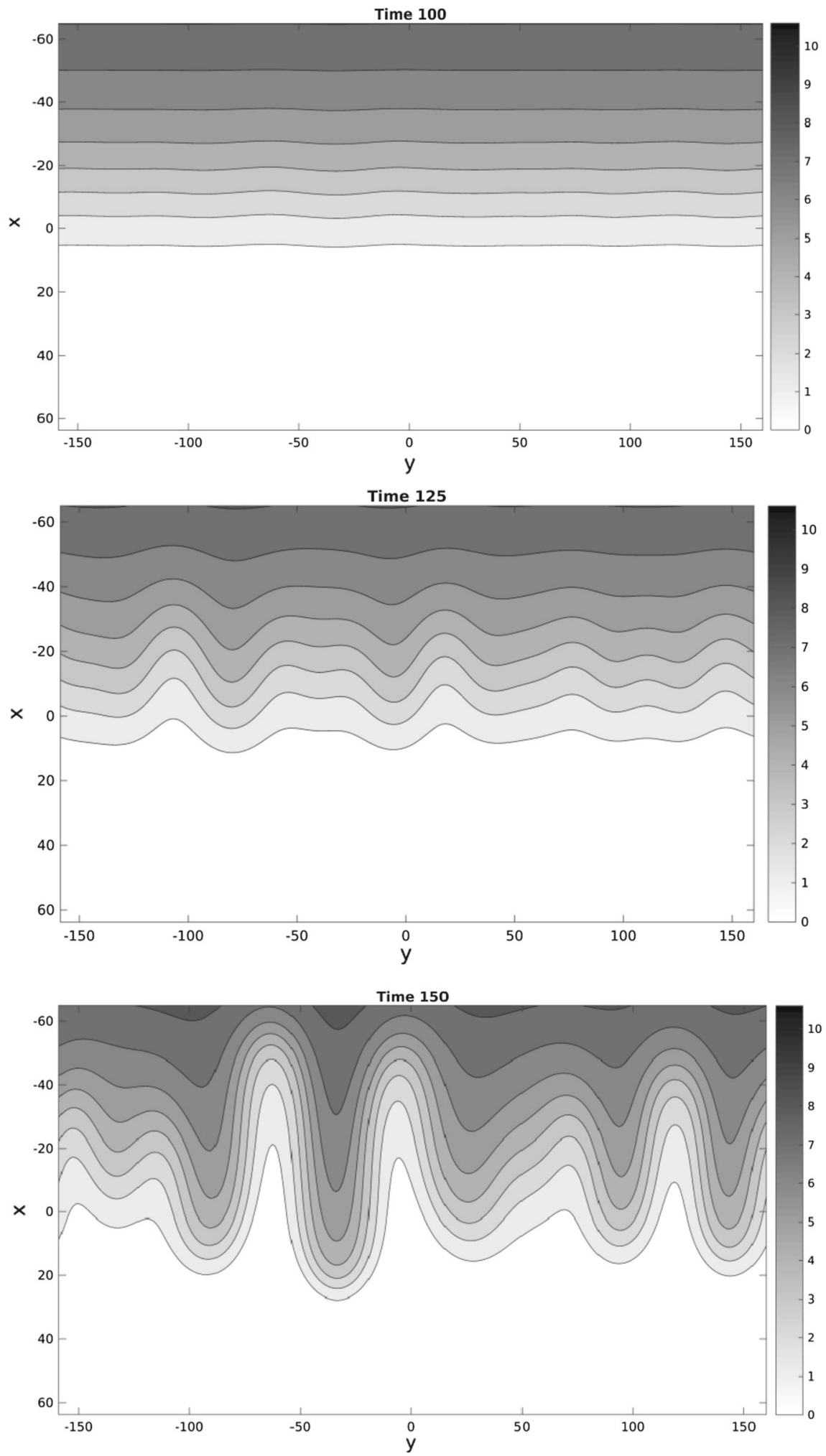

Fig. 3 Nonlinear simulations illustrating the evolution of the instability when $m=1 / 2$ in a domain $-160<y<160$ at times $T=100$, 125 and 150 


$$
+f_{\theta} G_{s}^{m}\left(\lambda_{m}+\frac{\eta}{\tau^{(1+m) / 4}}\right)=a_{\eta \eta}+a_{\theta \theta}
$$

We can expand the $G_{s}^{m}$ term in the large $\tau$ limit to obtain

$$
G_{s}^{m}\left(\lambda_{m}+\frac{\eta}{\tau^{(1+m) / 4}}\right)=G_{s}^{m}\left(\lambda_{m}\right)+\frac{\eta G_{s s}^{m}\left(\lambda_{m}\right)}{\tau^{(1+m) / 4}}+\frac{\eta^{2} G_{s s s}^{m}\left(\lambda_{m}\right)}{2 \tau^{(1+m) / 2}}+\frac{\eta^{3} G_{s s s s}^{m}\left(\lambda_{m}\right)}{6 \tau^{3(1+m) / 4}}+\frac{\eta^{4} G_{s s s s}^{m}\left(\lambda_{m}\right)}{24 \tau^{1+m}}+\cdots
$$

As $G_{s}^{m}$ is most negative at $s=\lambda_{m}$ this means that $G_{s s}^{m}\left(\lambda_{m}\right)=0$. We shall supress the writing of $\left(\lambda_{m}\right)$ as all of the derivatives of $G^{m}$ are evaluated at $s=\lambda_{m}$. Introducing

$\phi=\frac{1+m}{4}$

and subbing the expansion into Eq. (26) yields

$$
\begin{aligned}
& a_{\tau} \tau^{1-2 \phi}-\left[\frac{\lambda_{m}}{2 \tau^{\phi}}+\frac{q \eta}{\tau^{2 \phi}}\right] a_{\eta}-\frac{q \theta}{\tau^{2 \phi}} a_{\theta} \\
& \quad+f_{\theta}\left[G_{s}^{m}+\frac{\eta^{2} G_{s s s}^{m}}{2 \tau^{2 \phi}}+\frac{\eta^{3} G_{s s s s}^{m}}{6 \tau^{3 \phi}}+\frac{\eta^{4} G_{s s s s s}^{m}}{24 \tau^{4 \phi}}+\cdots\right]=a_{\eta \eta}+a_{\theta \theta} .
\end{aligned}
$$

To balance this equation, we introduce

$a(\eta, \theta, \tau)=\hat{a} J(\tau)+$ c.c. and $f(\eta, \theta, \tau)=\hat{f} J(\tau)+$ c.c.,

where c.c. denotes the complex conjugate parts and

$$
\begin{aligned}
& \hat{a}=a^{0}+\frac{a^{1}}{\tau^{\phi}}+\frac{a^{2}}{\tau^{2 \phi}}+\frac{a^{3}}{\tau^{3 \phi}}+\frac{a^{4}}{\tau^{4 \phi}}+\cdots, \\
& \hat{f}=f^{0}+\frac{f^{1}}{\tau^{\phi}}+\frac{f^{2}}{\tau^{2 \phi}}+\frac{f^{3}}{\tau^{3 \phi}}+\frac{f^{4}}{\tau^{4 \phi}}+\cdots, \\
& J(\tau)=\exp \left(\frac{\beta_{0}}{2 \phi} \tau^{2 \phi}+\frac{\beta_{1}}{\phi} \tau^{\phi}+\beta_{2} \ln (\tau)-\frac{\beta_{3}}{\phi} \tau^{-\phi}-\frac{\beta_{4}}{2 \phi} \tau^{-2 \phi}\right),
\end{aligned}
$$

where $a^{j}$ and $f^{j}$ are functions of $\eta$ and $\theta$ whilst $\beta_{j}$ are constants to be determined. Subbing these expressions into Eq. (24) and collecting terms with like powers of $\tau$ yields

$f_{\eta \eta}^{j}+f_{\theta \theta}^{j}=a_{\theta}^{j}$ for $0 \leq j \leq 4$.

Subbing these expressions into Eq. (27) and retaining powers of $\tau$ down to and including $-4 \phi$ yields

$$
\begin{aligned}
\left(a^{0}\right. & \left.+\frac{a^{1}}{\tau^{\phi}}+\frac{a^{2}}{\tau^{2 \phi}}+\frac{a^{3}}{\tau^{3 \phi}}+\frac{a^{4}}{\tau^{4 \phi}}+\cdots\right)\left(\beta_{0}+\frac{\beta_{1}}{\tau^{\phi}}+\frac{\beta_{2}}{\tau^{2 \phi}}+\frac{\beta_{3}}{\tau^{3 \phi}}+\frac{\beta_{4}}{\tau^{4 \phi}}\right)-\frac{\phi a^{1}}{\tau^{3 \phi}} \\
& -\frac{2 \phi a^{2}}{\tau^{4 \phi}}-\left[\frac{\lambda_{m}}{2 \tau^{\phi}}+\frac{q \eta}{\tau^{2 \phi}}\right]\left(a_{\eta}^{0}+\frac{a_{\eta}^{1}}{\tau^{\phi}}+\frac{a_{\eta}^{2}}{\tau^{2 \phi}}+\frac{a_{\eta}^{3}}{\tau^{3 \phi}}\right)-\frac{q \theta}{\tau^{2 \phi}}\left(a_{\theta}^{0}+\frac{a_{\theta}^{1}}{\tau^{\phi}}+\frac{a_{\theta}^{2}}{\tau^{2 \phi}}\right) \\
& +\left(f_{\theta}^{0}+\frac{f_{\theta}^{1}}{\tau^{\phi}}+\frac{f_{\theta}^{2}}{\tau^{2 \phi}}+\frac{f_{\theta}^{3}}{\tau^{3 \phi}}+\frac{f_{\theta}^{4}}{\tau^{4 \phi}}\right)\left[G_{s}^{m}+\frac{\eta^{2} G_{s s s}^{m}}{2 \tau^{2 \phi}}+\frac{\eta^{3} G_{s s s s}^{m}}{6 \tau^{3 \phi}}+\frac{\eta^{4} G_{s s s s s}^{m}}{24 \tau^{4 \phi}}\right] \\
= & a_{\eta \eta}^{0}+a_{\theta \theta}^{0}+\frac{a_{\eta \eta}^{1}+a_{\theta \theta}^{1}}{\tau^{\phi}}+\frac{a_{\eta \eta}^{2}+a_{\theta \theta}^{2}}{\tau^{2 \phi}}+\frac{a_{\eta \eta}^{3}+a_{\theta \theta}^{3}}{\tau^{3 \phi}}+\frac{a_{\eta \eta}^{4}+a_{\theta \theta}^{4}}{\tau^{4 \phi}}+\cdots
\end{aligned}
$$

Collecting terms with like powers of $\tau$ yields

$$
\begin{aligned}
& \beta_{0} a^{0}+G_{s}^{m} f_{\theta}^{0}=a_{\eta \eta}^{0}+a_{\theta \theta}^{0}, \\
& \beta_{0} a^{1}+\beta_{1} a^{0}-\frac{\lambda_{m}}{2} a_{\eta}^{0}+G_{s}^{m} f_{\theta}^{1}=a_{\eta \eta}^{1}+a_{\theta \theta}^{1}, \\
& \beta_{0} a^{2}+\beta_{1} a^{1}+\beta_{2} a^{0}-\frac{\lambda_{m}}{2} a_{\eta}^{1}-q \eta a_{\eta}^{0}
\end{aligned}
$$




$$
\begin{aligned}
& -q \theta a_{\theta}^{0}+G_{s}^{m} f_{\theta}^{2}+\frac{\eta^{2}}{2} G_{s s s}^{m} f_{\theta}^{0}=a_{\eta \eta}^{2}+a_{\theta \theta}^{2}, \\
& \beta_{0} a^{3}+\beta_{1} a^{2}+\beta_{2} a^{1}+\beta_{3} a^{0}-\phi a^{1}-\frac{\lambda_{m}}{2} a_{\eta}^{2}-q \eta a_{\eta}^{1} \\
& -q \theta a_{\theta}^{1}+G_{s}^{m} f_{\theta}^{3}+\frac{\eta^{2}}{2} G_{s s s}^{m} f_{\theta}^{1}+\frac{\eta^{3}}{6} G_{s s s s}^{m} f_{\theta}^{0}=a_{\eta \eta}^{3}+a_{\theta \theta}^{3}, \\
& \beta_{0} a^{4}+\beta_{1} a^{3}+\beta_{2} a^{2}+\beta_{3} a^{1}+\beta_{4} a^{0}-2 \phi a^{2} \\
& -\frac{\lambda_{m}}{2} a_{\eta}^{3}-q \eta a_{\eta}^{2}-q \theta a_{\theta}^{2}+G_{s}^{m} f_{\theta}^{4} \\
& +\frac{\eta^{2}}{2} G_{s s s}^{m} f_{\theta}^{2}+\frac{\eta^{3}}{6} G_{s s s s}^{m} f_{\theta}^{1}+\frac{\eta^{4}}{24} G_{s s s s}^{m} f_{\theta}^{0}=a_{\eta \eta}^{4}+a_{\theta \theta}^{4} .
\end{aligned}
$$

First, we notice in Eqs. (32), (33), and (34) that the coefficients are independent of $\eta$ and $\theta$ so we can seek a normal form solution given by

$\left[f^{0}, f^{1}, a^{0}, a^{1}\right]=\left[\mathrm{i} f_{0}, \mathrm{i} f_{1}, a_{0}, a_{1}\right] \mathrm{e}^{\mathrm{i} l \eta+\mathrm{i} \kappa \theta}$

where $\kappa$ and $l$ are the wavenumbers in the $\theta$ and $\eta$ directions and $a_{i}$ and $f_{i}$ are constants. Then Eq. (32) for $j=1$ and (33) become

$-\mathcal{N} f_{0}=\kappa a_{0}$ and $\beta_{0} a_{0}-\kappa f_{0} G_{s}^{m}=-a_{0} \mathcal{N}$, where $\mathcal{N}=\kappa^{2}+l^{2}$.

Hence, we obtain

$\beta_{0}=-\frac{\kappa^{2} G_{s}^{m}}{\mathcal{N}}-\mathcal{N}$

Second, Eq. (32) for $j=1$ and (34) become

$-\mathcal{N} f_{1}=\kappa a_{1}$ and $\beta_{0} a_{1}+\beta_{1} a_{0}-\frac{\lambda_{m}}{2} \mathrm{i} l a_{0}-\kappa G_{s}^{m} f_{1}=-a_{1} \mathcal{N}$.

Hence, we obtain

$\beta_{1}=\frac{\mathrm{i} l \lambda_{m}}{2}$.

Next we examine Eq. (32) for $j=2$ and (35). Although the coefficients of the terms involving $a^{2}$ and $f^{2}$ are independent of $\eta$ and $\theta$, there are terms including $\eta$ and $\theta$ in Eq. (35). Hence, to obtain a particular solution to this problem, we seek a solution of the form:

$f^{2}=\mathrm{i}\left(f_{2} \eta+f_{3} \eta^{2}+f_{4} \eta^{3}+f_{5} \theta^{2}\right) \mathrm{e}^{\mathrm{i} l \eta+\mathrm{i} \kappa \theta}$,

$a^{2}=\left(a_{2}+a_{3} \eta+a_{4} \eta^{2}+a_{5} \eta^{3}+a_{6} \theta+a_{7} \theta^{2}\right) \mathrm{e}^{\mathrm{i} l \eta+\mathrm{i} \kappa \theta}$

where $f_{j}$ and $a_{j}$ are constants. Subbing these expressions into Eq. (32) for $j=2$ and equating coefficients of powers of $\eta$ and $\theta$, we obtain

$a_{2}=\frac{2 \mathrm{i} l f_{2}+2 f_{3}}{\kappa}+\frac{2 l^{2} f_{5}}{\kappa^{3}}, \quad a_{3}=\frac{4 \mathrm{i} l f_{3}+6 f_{4}-\mathcal{N} f_{2}}{\kappa}$,

$a_{4}=\frac{6 \mathrm{i} l f_{4}-\mathcal{N} f_{3}}{\kappa}, \quad a_{5}=-\frac{\mathcal{N} f_{4}}{\kappa}, \quad a_{6}=\frac{2 \mathrm{i}\left(\kappa^{2}-l^{2}\right) f_{5}}{\kappa^{2}}, \quad a_{7}=-\frac{\mathcal{N} f_{5}}{\kappa}$.

Using Eq. (35) and equating coefficients of powers of $\eta$ and $\theta$, we obtain

$$
\begin{aligned}
& \frac{f_{3}}{f_{0}}=\frac{\kappa^{2} \mathcal{N} G_{s s s}^{m}}{8 l^{2} \mathcal{R}}+\frac{\kappa^{2} \mathcal{N}^{2} G_{s s s}^{m}}{2 \mathcal{R}^{2}}-\frac{q \mathcal{N}^{2}}{4 \mathcal{R}}, \quad \frac{f_{4}}{f_{0}}=-\frac{\mathrm{i} \kappa^{2} \mathcal{N} G_{s s s}^{m}}{12 l \mathcal{R}}, \quad \frac{f_{5}}{f_{0}}=-\frac{q \mathcal{N}^{2}}{4 \mathcal{S}}, \\
& \beta_{2}=\frac{4 \kappa^{2} l^{2} \mathcal{N} G_{s s s}^{m}}{\mathcal{R}^{2}}+\frac{G_{s s s}^{m}}{4 l^{2}}-\frac{G_{s s s}^{m}}{4 \mathcal{N}}-\frac{2 q \kappa^{2} \mathcal{N}}{\mathcal{S}}-\frac{2 q l^{2} \mathcal{N}}{\mathcal{R}}+\frac{2 i l f_{2}}{f_{0}}\left(1-\frac{\kappa^{2} G_{s}^{m}}{\mathcal{N}^{2}}\right),
\end{aligned}
$$


where

$\mathcal{R}=\mathcal{N}^{2}-G_{s}^{m} \kappa^{2}$ and $\mathcal{S}=\mathcal{N}^{2}+G_{s}^{m} l^{2}$.

Notice we have 1 free unknown $f_{2}$. However, the expression for $\beta_{2}$ is singular when $l=0$ and $\mathcal{S}=0$. We can remove the singularities at $l=0$ and $\mathcal{S}=0$ in $\beta_{2}$ by choosing

$\frac{f_{2}}{f_{0}}=\frac{\mathrm{i} G_{s s s}^{m} \mathcal{N}^{2}}{8 l^{3} \mathcal{R}}-\frac{\mathrm{i} q l \kappa^{2}}{\mathcal{S}}-\frac{\mathrm{i} q \kappa^{4}}{l \mathcal{R}}$

to obtain

$\beta_{2}=\frac{4 \kappa^{2} l^{2} \mathcal{N} G_{s s s}^{m}}{\mathcal{R}^{2}}-\frac{G_{s s s}^{m}}{4 \mathcal{N}}-\frac{2 q l^{2} \mathcal{N}}{\mathcal{R}}$.

Next we examine Eq. (32) for $j=3$ and (36). Although the coefficients of the terms involving $a^{3}$ and $f^{3}$ are independent of $\eta$ and $\theta$, there are terms including $\eta$ and $\theta$ in Eq. (36). Hence, to obtain a particular solution to this problem, we choose $f_{1}=0$ and seek a solution of the form

$f^{3}=\mathrm{i}\left(f_{6} \eta+f_{7} \eta^{2}+f_{8} \eta^{3}+f_{9} \eta^{4}\right) \mathrm{e}^{\mathrm{i} l \eta+\mathrm{i} \kappa \theta}$

$a^{3}=\left(a_{8}+a_{9} \eta+a_{10} \eta^{2}+a_{11} \eta^{3}+a_{12} \eta^{4}\right) \mathrm{e}^{\mathrm{i} l \eta+\mathrm{i} \kappa \theta}$,

where $f_{j}$ and $a_{j}$ are constants. Subbing these expressions into Eq. (32) for $j=3$ and equating coefficients of powers of $\eta$, we obtain

$$
\begin{aligned}
a_{8} & =\frac{2 \mathrm{i} l f_{6}+2 f_{7}}{\kappa}, \quad a_{9}=\frac{4 \mathrm{i} l f_{7}+6 f_{8}-\mathcal{N} f_{6}}{\kappa}, \\
a_{10} & =\frac{6 \mathrm{i} l f_{8}+12 f_{9}-\mathcal{N} f_{7}}{\kappa}, \quad a_{11}=\frac{8 \mathrm{il} f_{9}-\mathcal{N} f_{8}}{\kappa}, \quad a_{12}=-\frac{\mathcal{N} f_{9}}{\kappa} .
\end{aligned}
$$

Using Eq. (35) and equating coefficients of powers of $\eta$, we obtain

$$
\begin{aligned}
\frac{f_{7}}{f_{0}}= & \frac{\mathrm{i} \kappa^{2} \mathcal{N} G_{s s s}^{m}}{16 l^{3} \mathcal{R}}+\frac{\mathrm{i} l \kappa^{2} \mathcal{N}^{3} G_{s s s s}^{m}}{\mathcal{R}^{3}}-\frac{\mathrm{i} q \lambda_{m} \mathcal{N}^{4}}{16 l \mathcal{R}^{2}} \\
& +\frac{\mathrm{i} \lambda_{m} \kappa^{2} \mathcal{N}^{4} G_{s s s}^{m}}{4 l \mathcal{R}^{3}}+\frac{\mathrm{i} \lambda_{m} \kappa^{2}\left(\kappa^{2}-l^{2}\right) \mathcal{N}^{2} G_{s s s}^{m}}{16 l^{3} \mathcal{R}^{2}} \\
\frac{f_{8}}{f_{0}}= & \frac{\kappa^{2} \mathcal{N} G_{s s s s}^{m}}{24 l^{2} \mathcal{R}}+\frac{\kappa^{2} \mathcal{N}^{2} G_{s s s s}^{m}}{6 \mathcal{R}^{2}}+\frac{\lambda_{m} \kappa^{2} \mathcal{N}^{3} G_{s s s}^{m}}{48 l^{2} \mathcal{R}^{2}}, \quad \frac{f_{9}}{f_{0}}=-\frac{\mathrm{i} \kappa^{2} \mathcal{N} G_{s s s s}^{m}}{48 l \mathcal{R}} \\
\beta_{3}= & \frac{2 \mathrm{i} l f_{6}}{f_{0}}\left(1-\frac{\kappa^{2} G_{s}^{m}}{\mathcal{N}^{2}}\right)+\mathrm{i} \kappa^{2}\left(\frac{8 l^{3} \mathcal{N}^{2}}{\mathcal{R}^{3}}-\frac{2 l \mathcal{N}}{\mathcal{R}^{2}}+\frac{1}{8 l^{3} \mathcal{N}}\right) G_{s s s s}^{m} \\
& +\mathrm{i} \lambda_{m}\left(\frac{2 l \kappa^{2} \mathcal{N}^{3}}{\mathcal{R}^{3}}-\frac{\kappa^{2} \mathcal{N}\left(3 l^{2}-\kappa^{2}\right)}{2 l \mathcal{R}^{2}}+\frac{3 \kappa^{4}+l^{4}}{16 l^{3} \mathcal{R}}\right) G_{s s s}^{m} \\
& +\mathrm{i} \lambda_{m} q\left(\frac{3 l^{4}+2 l^{2} \kappa^{2}-5 \kappa^{4}}{8 l \mathcal{R}}-\frac{l \kappa^{2}}{2 \mathcal{S}}-\frac{l \mathcal{N}^{3}}{2 \mathcal{R}^{2}}\right)
\end{aligned}
$$

Notice that $\beta_{3}$ is singular at $l=0$ and $\mathcal{S}=0$. We can use partial fractions in the variable $l$ to identify the singular terms at $l=0$ and $\mathcal{S}=0$ and then remove these singularities in $\beta_{3}$ by choosing

$$
\begin{aligned}
\frac{f_{6}}{f_{0}}=\frac{\left(\kappa^{2}+G_{s}^{m}\right) G_{s s s s}^{m}}{16 l^{2} \mathcal{I}^{2}}-\frac{\kappa^{2} G_{s s s s}^{m}}{16 l^{4} \mathcal{I}}-\frac{3 \lambda_{m} \kappa^{4} G_{s s s}^{m}}{32 l^{4} \mathcal{I}^{2}}+\frac{\lambda_{m} \kappa^{2} G_{s s s}^{m}\left(3 G_{s}^{m}-\kappa^{2}\right)}{16 l^{2} \mathcal{I}^{3}} \\
-\frac{\left(\mathcal{N}\left(G_{s}^{m}\right)^{2}-\left(6 \kappa^{2}+4 l^{2}\right) \kappa^{2} G_{s}^{m}+\left(5 \kappa^{2}+3 l^{2}\right) \kappa^{4}\right) G_{s}^{m} G_{s s s s}^{m}}{16 \kappa^{2} \mathcal{I}^{3} \mathcal{R}} \\
-\frac{\lambda_{m} \kappa^{2}\left(3 G_{s}^{m}+5 \kappa^{2}+4 l^{2}\right) G_{s}^{m} G_{s s s}^{m}}{32 \mathcal{I}^{3} \mathcal{R}}-\frac{q \lambda_{m} \kappa^{2} \mathcal{N}}{4 G_{s}^{m} \mathcal{S}}+\frac{5 q \lambda_{m} \kappa^{4}}{16 l^{2} \mathcal{I}^{2}}
\end{aligned}
$$




$$
+\frac{\lambda_{m} q \kappa^{4}\left(\left(6 \kappa^{2}+l^{2}\right)\left(G_{s}^{m}\right)^{3}+\left(2 \kappa^{2}+7 l^{2}\right) \kappa^{2}\left(G_{s}^{m}\right)^{2}-12 \kappa^{4} \mathcal{N} G_{s}^{m}+4 \kappa^{6} \mathcal{N}\right)}{16 \kappa^{2} G_{s}^{m} \mathcal{I}^{3} \mathcal{R}}
$$

where $\mathcal{I}=\kappa^{2}-G_{s}^{m}$, to yield

$$
\begin{aligned}
\beta_{3}=- & \frac{\mathrm{i} \lambda_{m} q l \kappa^{2} \mathcal{N} G_{s}^{m}}{2 \mathcal{R}^{2}}+\frac{\mathrm{i} \lambda_{m} q l\left(4 \kappa^{2}+G_{s}^{m}\right)\left(2 \kappa^{2}+l^{2}\right)}{8 \mathcal{R} \mathcal{I}}+\frac{8 \mathrm{i} l^{3} \kappa^{4} G_{s}^{m} G_{s s s}^{m}}{\mathcal{R}^{3}} \\
& +\frac{2 \mathrm{i} l \kappa^{2}\left(3 l^{2}-\kappa^{2}\right) G_{s s s s}^{m}}{\mathcal{R}^{2}}+\frac{\mathrm{i} l G_{s s s s}^{m}}{8 \kappa^{2} \mathcal{N}}-\frac{\mathrm{i} l \lambda_{m} \kappa^{2} G_{s}^{m}\left(l^{2}+2 \kappa^{2}\right) G_{s s s}^{m}}{2 \mathcal{I} \mathcal{R}^{2}} \\
& +\frac{2 \mathrm{i} l \lambda_{m} \kappa^{4} \mathcal{N} G_{s}^{m} G_{s s s}^{m}}{\mathcal{R}^{3}}-\frac{\mathrm{i} \lambda_{m} l\left(6 \kappa^{4}+2 l^{2} \kappa^{2}-\kappa^{2} G_{s}^{m}-\left(G_{s}^{m}\right)^{2}\right) G_{s s s}^{m}}{16 \mathcal{I}^{2} \mathcal{R}} .
\end{aligned}
$$

Finally, we solve Eqs. (32) for $j=4$ and (37). To obtain a particular solution to this problem, we seek a solution of the form:

$$
\begin{aligned}
f^{4}=\mathrm{i}[ & \left.f_{10} \eta+f_{11} \eta^{3}+f_{12} \eta^{4}+f_{13} \eta^{5}+f_{14} \eta^{6}+\theta\left(f_{15} \eta+f_{16} \eta^{2}+f_{17} \eta^{3}\right)\right] \mathrm{e}^{\mathrm{i} l \eta+\mathrm{i} \kappa \theta} \\
& +\mathrm{i}\left[\theta^{2}\left(f_{18} \eta+f_{19} \eta^{2}+f_{20} \eta^{3}\right)+f_{21} \theta^{3}+f_{22} \theta^{4}\right] \mathrm{e}^{\mathrm{i} l \eta+\mathrm{i} \kappa \theta}, \\
a^{4}=\left[a_{13}+a_{14} \eta+a_{15} \eta^{2}+a_{16} \eta^{3}+a_{17} \eta^{4}+a_{18} \eta^{5}+a_{19} \eta^{6}\right] \mathrm{e}^{\mathrm{i} l \eta+\mathrm{i} \kappa \theta} & +\left[\theta\left(a_{20}+a_{21} \eta+a_{22} \eta^{2}+a_{23} \eta^{3}\right)+\theta^{2}\left(a_{24}+a_{25} \eta+a_{26} \eta^{2}+a_{27} \eta^{3}\right)\right] \mathrm{e}^{\mathrm{i} l \eta+\mathrm{i} \kappa \theta} \\
+ & {\left[a_{28} \theta^{3}+a_{29} \theta^{4}\right] \mathrm{e}^{\mathrm{i} l \eta+\mathrm{i} \kappa \theta}, }
\end{aligned}
$$

where $f_{j}$ and $a_{j}$ are constants. We find that $\beta_{4}$ has singularities at $l=0$ and $\mathcal{S}=0$, which can be removed by choosing $f_{10}$ appropriately. We expect the system to be most unstable when $l=0$, and in this limit, we find that

$\beta_{0}=-G_{s}^{m}-\kappa^{2}, \quad \beta_{1}=\beta_{3}=0, \quad$ and $\beta_{2}=-\frac{G_{s s s}^{m}}{4 \kappa^{2}}$,

and

$$
\begin{aligned}
& \beta_{4}=\frac{\left(32 \kappa^{10}-120 \kappa^{8} G_{s}^{m}+160 \kappa^{6}\left(G_{s}^{m}\right)^{2}-83 \kappa^{4}\left(G_{s}^{m}\right)^{3}\right) G_{s s s s s}^{m}}{2 \mathcal{I}^{4}\left(G_{s}^{m}\right)^{3}} \\
& +\frac{\left(58 \kappa^{10} G_{s}^{m}+27 \kappa^{8}\left(G_{s}^{m}\right)^{2}+2 \kappa^{4}\left(G_{s}^{m}\right)^{4}-2 \kappa^{2}\left(G_{s}^{m}\right)^{5}+3\left(G_{s}^{m}\right)^{6}\right) G_{s s s s}^{m}}{16 \kappa^{8} \mathcal{I}^{4}} \\
& +\frac{\lambda_{m}\left(192 \kappa^{10}-688 G_{s}^{m} \kappa^{8}+868\left(G_{s}^{m}\right)^{2} \kappa^{6}-413 \kappa^{4}\left(G_{s}^{m}\right)^{3}\right) G_{s s s s}^{m}}{32 \mathcal{I}^{4}\left(G_{s}^{m}\right)^{3}} \\
& +\frac{\lambda_{m}\left(42 \kappa^{8} G_{s}^{m}+24 \kappa^{6}\left(G_{s}^{m}\right)^{2}-11 \kappa^{4}\left(G_{s}^{m}\right)^{3}-5 \kappa^{2}\left(G_{s}^{m}\right)^{4}-9\left(G_{s}^{m}\right)^{5}\right) G_{s s s s}^{m}}{32 \kappa^{6} \mathcal{I}^{4}} \\
& +\frac{\left(1024 \kappa^{12}-3824 \kappa^{10} G_{s}^{m}+5092 \kappa^{8}\left(G_{s}^{m}\right)^{2}-2609 \kappa^{6}\left(G_{s}^{m}\right)^{3}\right)\left(G_{s s S}^{m}\right)^{2}}{4 \mathcal{I}^{4}\left(G_{s}^{m}\right)^{5}} \\
& +\frac{\left(2196 \kappa^{6}+1558 \kappa^{4} G_{s}^{m}+10 \kappa^{2}\left(G_{s}^{m}\right)^{2}-687\left(G_{s}^{m}\right)^{3}\right)\left(G_{s s s}^{m}\right)^{2}}{64 \kappa^{2} \mathcal{I}^{4} G_{s}^{m}} \\
& +\frac{\left(-872\left(G_{s}^{m}\right)^{3} \kappa^{6}-716\left(G_{s}^{m}\right)^{4} \kappa^{4}-246\left(G_{s}^{m}\right)^{5} \kappa^{2}-171\left(G_{s}^{m}\right)^{6}\right)\left(G_{s s s}^{m}\right)^{2}}{64 \kappa^{10} \mathcal{I}^{4}} \\
& +\frac{q\left(-480 \kappa^{12}+1644 \kappa^{10} G_{s}^{m}-1824 \kappa^{8}\left(G_{s}^{m}\right)^{2}+436 \kappa^{6}\left(G_{s}^{m}\right)^{3}\right) G_{s s s}^{m}}{\mathcal{I}^{4}\left(G_{s}^{m}\right)^{4}} \\
& +\frac{q\left(12630 \kappa^{6}-4875 \kappa^{4} G_{s}^{m}-780 \kappa^{2}\left(G_{s}^{m}\right)^{2}+346\left(G_{s}^{m}\right)^{3}\right) G_{s s s}^{m}}{32 \kappa^{2} \mathcal{I}^{4}} \\
& +\frac{q\left(126 \kappa^{2}\left(G_{s}^{m}\right)^{4}+105\left(G_{s}^{m}\right)^{5}\right) G_{s s S}^{m}}{32 \kappa^{6} \mathcal{I}^{4}}+\frac{\left(4 \kappa^{10}-14 \kappa^{8} G_{s}^{m}+17 \kappa^{6}\left(G_{s}^{m}\right)^{2}\right) G_{s S S}^{m}}{\mathcal{I}^{4}\left(G_{s}^{m}\right)^{3}} \\
& +\frac{\left(-238 \kappa^{8}+23 \kappa^{6} G_{s}^{m}+20 \kappa^{4}\left(G_{s}^{m}\right)^{2}-14 \kappa^{2}\left(G_{s}^{m}\right)^{3}-6\left(G_{s}^{m}\right)^{4}\right) G_{s s s}^{m}}{32 \kappa^{4} \mathcal{I}^{4}}
\end{aligned}
$$




$$
\begin{aligned}
& -\frac{9\left(G_{s}^{m}\right)^{5} G_{s s s}^{m}}{32 \kappa^{6} \mathcal{I}^{4}}+\frac{\lambda_{m}^{2}\left(-32 \kappa^{8}+108 \kappa^{6} G_{s}^{m}-115 \kappa^{4}\left(G_{s}^{m}\right)^{2}\right) G_{s s s}^{m}}{64 \mathcal{I}^{4}\left(G_{s}^{m}\right)^{2}} \\
& +\frac{\lambda_{m}^{2}\left(-\kappa^{6} G_{s}^{m}+31 \kappa^{4}\left(G_{s}^{m}\right)^{2}+16 \kappa^{2}\left(G_{s}^{m}\right)^{3}+9\left(G_{s}^{m}\right)^{4}\right) G_{s s s}^{m}}{64 \kappa^{4} \mathcal{I}^{4}} \\
& +\frac{q\left(2 \kappa^{8}-5 \kappa^{6} G_{s}^{m}+5 \kappa^{4}\left(G_{s}^{m}\right)^{2}-3 \kappa^{2}\left(G_{s}^{m}\right)^{3}+\left(G_{s}^{m}\right)^{4}\right)}{4 \kappa^{2} \mathcal{I}^{4}} \\
& +\frac{q \lambda_{m}^{2}\left(\left(G_{s}^{m}\right)^{3}+\kappa^{2}\left(G_{s}^{m}\right)^{2}-11 \kappa^{4} G_{s}^{m}+3 \kappa^{6}\right)}{32 \kappa^{2} \mathcal{I}^{3}} \\
& +\frac{q^{2}\left(384 \kappa^{10}-1520 \kappa^{8} G_{s}^{m}+2192 \kappa^{6}\left(G_{s}^{m}\right)^{2}-1297 \kappa^{4}\left(G_{s}^{m}\right)^{3}\right)}{16 \mathcal{I}^{4}\left(G_{s}^{m}\right)^{2}} \\
& +\frac{q^{2}\left(168 \kappa^{6}\left(G_{s}^{m}\right)^{2}+130 \kappa^{4}\left(G_{s}^{m}\right)^{3}-56 \kappa^{2}\left(G_{s}^{m}\right)^{4}-\left(G_{s}^{m}\right)^{5}\right)}{16 \kappa^{4} \mathcal{I}^{4}} .
\end{aligned}
$$

Here, we shall use $(1 / \hat{A})(\mathrm{d} \hat{A} / \mathrm{d} \tau)$ as the definition of the growth rate. We recall that $\hat{A}=a=\hat{a} J+\overline{\hat{a}} \bar{J}$,

where bars are used to denote the complex conjugate. Now as $\beta_{0}$ to $\beta_{4}$ are real, $J=\bar{J}$ and so $\hat{A}=(\hat{a}+\overline{\hat{a}}) J$.

Thus,

$$
\begin{aligned}
\frac{1}{\hat{A}} \frac{\mathrm{d} \hat{A}}{\mathrm{~d} \tau}= & \frac{1}{(\hat{a}+\overline{\hat{a}}) J}\left((\hat{a}+\overline{\hat{a}}) \frac{\mathrm{d} J}{\mathrm{~d} \tau}+\frac{\mathrm{d}(\hat{a}+\overline{\hat{a}})}{\mathrm{d} \tau} J\right)=\frac{1}{J} \frac{\mathrm{d} J}{\mathrm{~d} \tau}+\frac{1}{\hat{a}+\overline{\hat{a}}} \frac{\mathrm{d}(\hat{a}+\overline{\hat{a}})}{\mathrm{d} \tau} \\
= & \beta_{0} \tau^{2 \phi-1}+\beta_{1} \tau^{\phi-1}+\beta_{2} \tau^{-1}+\beta_{3} \tau^{-\phi-1}+\beta_{4} \tau^{-2 \phi-1}+\cdots \\
& -\frac{1}{a^{0}+\overline{a^{0}}+\left(a^{1}+\overline{a^{1}}\right) \tau^{-\phi}+\left(a^{2}+\overline{a^{2}}\right) \tau^{-2 \phi}+\left(a^{3}+\overline{a^{3}}\right) \tau^{-3 \phi}+\cdots} \\
& \times \frac{\phi}{\tau}\left[\frac{a^{1}+\overline{a^{1}}}{\tau^{\phi}}+\frac{2\left(a^{2}+\overline{a^{2}}\right)}{\tau^{2 \phi}}+\frac{3\left(a^{3}+\overline{a^{3}}\right)}{\tau^{3 \phi}}+\frac{4\left(a^{4}+\overline{a^{4}}\right)}{\tau^{4 \phi}}+\cdots\right] .
\end{aligned}
$$

Including terms up to $\tau^{-2 \phi-1}$ reduces this to

$$
\begin{aligned}
\frac{1}{\hat{A}} \frac{\mathrm{d} \hat{A}}{\mathrm{~d} \tau}=\beta_{0} \tau^{2 \phi-1}+\beta_{1} \tau^{\phi-1}+\beta_{2} \tau^{-1}+\beta_{3} \tau^{-\phi-1}+\beta_{4} \tau^{-2 \phi-1} & -\phi \tau^{-1}\left[a^{0}+\overline{a^{0}}+\frac{a^{1}+\overline{a^{1}}}{\tau^{\phi}}\right]^{-1}\left[\frac{a^{1}+\overline{a^{1}}}{\tau^{\phi}}+\frac{2 a^{2}+2 \overline{a^{2}}}{\tau^{2 \phi}}\right] \\
= & \beta_{0} \tau^{2 \phi-1}+\beta_{1} \tau^{\phi-1}+\beta_{2} \tau^{-1}+\beta_{3} \tau^{-\phi-1}+\beta_{4} \tau^{-2 \phi-1} \\
& \quad-\phi \tau^{-1}\left(a^{0}+\overline{a^{0}}\right)^{-1}\left[1-\frac{a^{1}+\overline{a^{1}}}{\left(a^{0}+\overline{a^{0}}\right) \tau^{\phi}}\right]\left[\frac{a^{1}+\overline{a^{1}}}{\tau^{\phi}}+\frac{2 a^{2}+2 \overline{a^{2}}}{\tau^{2 \phi}}\right] \\
= & \beta_{0} \tau^{2 \phi-1}+\beta_{1} \tau^{\phi-1}+\beta_{2} \tau^{-1}+\beta_{3} \tau^{-\phi-1}+\beta_{4} \tau^{-2 \phi-1} \\
& \quad-\phi \tau^{-1}\left[\frac{a^{1}+\overline{a^{1}}}{\left(a^{0}+\overline{a^{0}}\right) \tau^{\phi}}+\frac{2 a^{2}+2 \overline{a^{2}}}{\left(a^{0}+\overline{a^{0}}\right) \tau^{2 \phi}}-\frac{\left(a^{1}+\overline{a^{1}}\right)^{2}}{\left(a^{0}+\overline{a^{0}}\right)^{2} \tau^{2 \phi}}\right] .
\end{aligned}
$$

Notice here we have taken $f_{1}=0$ and so $a^{1}=0$, and $\beta_{1}=\beta_{3}=0$. Subbing in $\phi=(m+1) / 4$ yields

$$
\frac{1}{\hat{A}} \frac{\mathrm{d} \hat{A}}{\mathrm{~d} \tau}=\beta_{0} \tau^{(m-1) / 2}+\beta_{2} \tau^{-1}+\left(\beta_{4}-\frac{2 \phi\left(a^{2}+\overline{a^{2}}\right)}{a^{0}+\overline{a^{0}}}\right) \tau^{-(m+3) / 2} .
$$


For large times $\tau$, we can expect long waves, i.e. as $\tau \rightarrow \infty, \kappa \rightarrow 0$; hence, as $\kappa \rightarrow 0$ we have $\beta_{4} \rightarrow \beta_{4}^{L}$ where

$$
\begin{aligned}
\beta_{4}^{L}=- & \frac{171\left(G_{s}^{m}\right)^{2}\left(G_{s s s}^{m}\right)^{2}}{64 \kappa^{10}}+\frac{6 G_{s s s s s}^{m}\left(G_{s}^{m}\right)^{2}-465\left(G_{s s s}^{m}\right)^{2} G_{s}^{m}}{32 \kappa^{8}}-\frac{601\left(G_{s s s}^{m}\right)^{2}}{4 G_{s}^{m} \kappa^{4}}-\frac{q^{2} G_{s}^{m}}{16 \kappa^{4}} \\
& +\frac{20 G_{s s s s}^{m} G_{s}^{m}-9 \lambda_{m} G_{s s s s}^{m} G_{s}^{m}+(105 q-9) G_{s s s}^{m} G_{s}^{m}-1705\left(G_{s s s}^{m}\right)^{2}}{32 \kappa^{6}} \\
& +\frac{96 G_{s s s s}^{m}-82 \lambda_{m} G_{s s s s}^{m}+\left(9 \lambda_{m}^{2}+1092 q-84\right) G_{s s s}^{m}}{64 \kappa^{4}}+\cdots
\end{aligned}
$$

As we are interested in the maximum growth rate around $\eta=0$ and $\theta=0$, we substitute $\theta=\eta=0$ to obtain

$\frac{a^{2}+\overline{a^{2}}}{a^{0}+\overline{a^{0}}}=\frac{q}{2 \kappa^{2}}-\frac{\kappa^{2} \mathcal{N} G_{s s s}^{m}}{\mathcal{R}^{2}}+\frac{G_{s s s}^{m}}{4 \mathcal{R}}-\frac{q l^{2} G_{s}^{m}}{2 \kappa^{2} \mathcal{S}}+\frac{q \mathcal{N}\left(G_{s}^{m}-4 \kappa^{2}\right)}{2 G_{s}^{m}}\left(\frac{1}{\mathcal{R}}-\frac{1}{\mathcal{S}}\right)$.

Then, when $l=0$, we have

$\frac{a^{2}+\overline{a^{2}}}{a^{0}+\overline{a^{0}}}=\frac{-G_{s s s}^{m}\left(3 \kappa^{2}+G_{s}^{m}\right)+6 q \kappa^{2}\left(\kappa^{2}-G_{s}^{m}\right)}{4 \kappa^{2}\left(\kappa^{2}-G_{s}^{m}\right)^{2}}$.

Expanding this about $\kappa=0$ we obtain

$\frac{a^{2}+\overline{a^{2}}}{a^{0}+\overline{a^{0}}} \rightarrow-\frac{G_{s s s}^{m}}{4 G_{s}^{m} \kappa^{2}}+\frac{3 q}{2 G_{s}^{m}}-\frac{5 G_{s s}^{m}}{4\left(G_{s}^{m}\right)^{2}}+\left(\frac{3 q}{2\left(G_{s}^{m}\right)^{2}}-\frac{9 G_{s s}^{m}}{4\left(G_{s}^{m}\right)^{3}}\right) \kappa^{2}+\cdots$

As the above term is only order $\kappa^{-2}$, whilst $\beta_{4}^{L}$ has a term of order $\kappa^{-10}$, the above term does not play a significant role in the instability. Hence, by retaining the most dominant terms, we have obtained

$\frac{1}{\hat{A}} \frac{\mathrm{d} \hat{A}}{\mathrm{~d} \tau}=\frac{-G_{s}^{m}-\kappa^{2}}{\tau^{(1-m) / 2}}-\frac{G_{s s s}^{m}}{4 \kappa^{2} \tau}+\beta_{4}^{L} \tau^{-(m+3) / 2}$.

To obtain the maximum growing wavenumber we set the derivative in $\kappa$ of the growth rate in Eq. (51) equal to zero. For large $\tau$ this yields the approximation

$\kappa_{\max }=\frac{(855)^{\frac{1}{12}}\left(-G_{s s s}^{m} G_{s}^{m}\right)^{\frac{1}{6}}}{\sqrt{2} \tau^{(m+1) / 12}}+\frac{8 G_{s}^{m} G_{s s s s}^{m}+(\sqrt{95}-620)\left(G_{s s s}^{m}\right)^{2}}{12 \sqrt{6}(95)^{\frac{3}{4}} \sqrt{-G_{s}^{m}}\left(G_{s s s}^{m}\right)^{3 / 2} \tau^{(m+1) / 4}}+\cdots$

Subbing the maximum growing wavenumber from Eq. (52) into Eq. (51), we obtain the maximum growth rate:

$$
\begin{aligned}
\max \left[\frac{1}{\hat{A}} \frac{\mathrm{d} \hat{A}}{\mathrm{~d} \tau}\right]= & -\frac{G_{s}^{m}}{\tau^{(1-m) / 2}}+\frac{3^{4 / 3} 95^{1 / 6}\left[-G_{s s s}^{m} G_{s}^{m}\right]^{1 / 3}}{5 \tau^{(2-m) / 3}} \\
& -\frac{2 G_{s}^{m} G_{s s s s s}^{m}+(\sqrt{95}-155)\left(G_{s s s}^{m}\right)^{2}}{2(3)^{1 / 3}(95)^{2 / 3}\left(G_{s s s}^{m}\right)^{4 / 3}\left(-G_{s}^{m}\right)^{1 / 3} \tau^{(5-m) / 6}}+\cdots
\end{aligned}
$$

In the next section, we shall attempt to numerically verify these scalings by performing a numerical linear stability analysis. One notes that this is the maximum growing wavenumber in the $\theta$ coordinate, using $Y=\tau^{(1-m) / 4} \theta$, means that the maximum growing wavenumber in the $Y$ coordinate is $k_{\max }=\kappa_{\max } / \tau^{(1-m) / 4}$, i.e.

$k_{\max }=\frac{(855)^{\frac{1}{12}}\left(G_{s s s}^{m}\left|G_{s}^{m}\right|\right)^{\frac{1}{6}}}{\sqrt{2} \tau^{(2-m) / 6}}+\frac{8 G_{s}^{m} G_{s s s s s}^{m}+(\sqrt{95}-620)\left(G_{s s s}^{m}\right)^{2}}{12 \sqrt{6}(95)^{\frac{3}{4}} \sqrt{-G_{s}^{m}}\left(G_{s s s}^{m}\right)^{3 / 2} \sqrt{\tau}}+\cdots$

One notes that $2 G_{s s}^{m}+s G_{s}^{m}-m G^{m}=0$ so $2 G_{s s s}^{m}+s G_{s s}^{m}+(1-m) G_{s}^{m}=0,2 G_{s s s s}^{m}+s G_{s s s}^{m}+(2-m) G_{s s}^{m}=0$ and $2 G_{s s s s}^{m}+s G_{s s s}^{m}+(3-m) G_{s s s}^{m}=0$. Hence, at $s=\lambda_{m}$ we have $G_{s s}^{m}=0$ so that

$G_{s s s}^{m}=\frac{m-1}{2} G_{s}^{m}, \quad G_{s s s s}^{m}=\frac{(1-m) \lambda_{m}}{4} G_{s}^{m}, \quad G_{s s s s}^{m}=\frac{m-1}{8} G_{s}^{m}\left(6-2 m-\lambda_{m}^{2}\right)$. 


\section{Numerical linear stability}

Using a scaling analysis for large times, we have found that asymptotically the perturbations of the instability do not grow like $\mathrm{e}^{\sigma T}$ when $m<1$. To determine the validity of these results we shall perform a numerical stability analysis to examine how the perturbations grow in time by assuming normal form expansions in the $Y$-direction

$[\psi, A]=[0, \tilde{A}(X, T)]+\epsilon[f(X, T), \hat{A}(X, T)] \mathrm{e}^{\mathrm{i} k Y}$,

where $k$ is the wavenumber. Subbing these expressions into Eqs. (9) and (10) and linearising in $\epsilon$, then collecting terms in $\epsilon$ yields

$$
\begin{aligned}
& \tilde{A}_{T}=\tilde{A}_{X X}, \\
& f_{X X}-k^{2} f=\hat{A}, \\
& \hat{A}_{T}-k^{2} f \tilde{A}_{X}=\hat{A}_{X X}-k^{2} \hat{A} .
\end{aligned}
$$

In order to obtain an instability, we introduce small pertubations in the initial conditions for $\hat{A}$ and $f$, and so in this section we use the following initial conditions:

$$
\begin{aligned}
& \tilde{A}(X, 0)=(-X)^{m} H(-X), \\
& \hat{A}(X, 0)=f(X, 0)=0.0001 \exp (-|X| / 10) .
\end{aligned}
$$

To carry out this numerical stability analysis, we truncate the infinite domain to the finite domain $|X|<h$ where $h$ is chosen to be sufficiently large that it does not affect the solutions obtained. Thus, we impose the following boundary conditions:

$$
\begin{aligned}
& \tilde{A}=h^{m} \text { and } f=\hat{A}=0 \quad \text { on } \quad X=-h, \\
& \tilde{A}=f=\hat{A}=0 \quad \text { on } \quad X=h .
\end{aligned}
$$

This coupled system of PDEs is numerically solved in time $T$ for different values of the wavenumber $k$ using the built in function pdepe in Matlab. Typically 4000 non-uniformally distributed spatial points were used. To ensure that the results were independent of the domain size $h$, typically $h$ was chosen to be greater than $8 \pi / k$. For each wavenumber investigated, we determine the growth rate of the perturbations using

$$
\frac{1}{\hat{A}} \frac{\mathrm{d} \hat{A}}{\mathrm{~d} T}
$$

at the spatial location where $\mathrm{d} \hat{A} / \mathrm{d} T$ is greatest. To evaluate $\mathrm{d} \hat{A} / \mathrm{d} T$, we use finite differences at two time steps separated in time by $\Delta T=10^{-4}$. To determine the maximum value of $(1 / \hat{A})(\mathrm{d} \hat{A} / \mathrm{d} T)$ at a given time $T$, we examine different wavenumbers $k$.

In Fig. 4a, we present log-log plots of the maximum growth rate with time $T$ for various values of $m$. In Fig. 4a, we see that there is good agreement between the numerically obtained maximum growth rate, the solid lines, and the large time asymptotic solution (53), the dashed curves. In Fig. 4b, we present $\log -\log$ plots of $T^{(1-m) / 2}$ multiplied by the difference between the maximum growth rate and the first term of the large time asymptotic growth rate in Eq. (53), with time $T$ for various $m$. In Fig. $4 \mathrm{~b}$ the dotted lines are straight lines fitted to the large time numerical solution. In Table 2, we represent the large time numerically obtained trends for $-(1 / \hat{A})(\mathrm{d} \hat{A} / \mathrm{d} T)-G_{s}^{m}\left(\lambda_{m}\right) T^{(m-1) / 2}$ for different values of $m$. Using Table 2, we can obtain the estimate

$\frac{1}{\hat{A}} \frac{\mathrm{d} \hat{A}}{\mathrm{~d} T}+\frac{G_{s}^{m}\left(\lambda_{m}\right)}{T^{(1-m) / 2}} \sim T^{0.367 m-0.658}$,

which has similar powers to those predicted by the linear stability theory, namely $T^{(m-2) / 3}$.

In Fig. 5, we present $\log -\log$ plots of the maximum growth rate with time $T$ for various values of $m$. In Fig. 5, we see that the agreement is not as good between the numerically obtained maximum growing wavenumber, the solid lines, and the large time asymptotic solution (54), the dashed curves. 
(a)

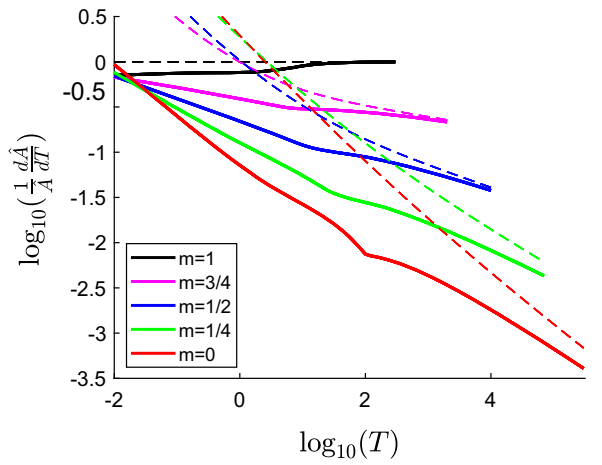

(b)

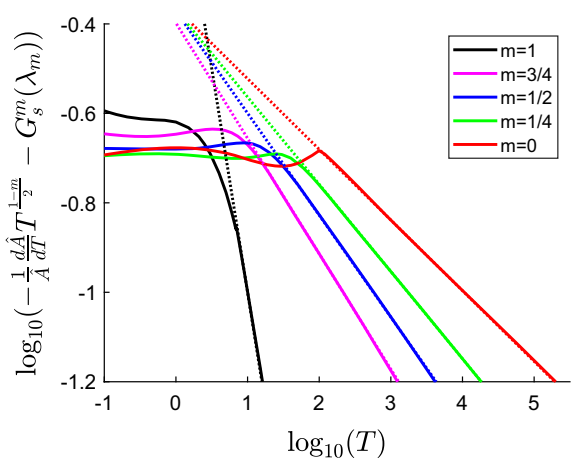

Fig. $4 \mathrm{Log}-\log$ plots of a the maximum growth rate, $(1 / \tilde{A})(d \tilde{A} / d T)$, and $\mathbf{b} T^{(1-m) / 2}$ multiplying the difference between the maximum growth rate and the first term of the large time asymptotic growth rate in Eq. (53), with time $T$ for $m=0,1 / 4,1 / 2,3 / 4$ and $m=1$. In a the dashed curves are the large time asymptotic solution given by Eq. (53) and in $\mathbf{b}$ the dotted lines are straight lines fitted to the large time numerical solution

Table 2 Large time numerical trends for $-(1 / \hat{A})(\mathrm{d} \hat{A} / \mathrm{d} T)-G_{s}^{m}\left(\lambda_{m}\right) T^{(m-1) / 2}$ for different values of $m$

\begin{tabular}{ll}
\hline$m$ & $-(1 / \hat{A})(\mathrm{d} \hat{A} / \mathrm{d} T)-G_{s}^{m}\left(\lambda_{m}\right) T^{(m-1) / 2}$ \\
\hline 0 & $0.432 T^{-0.658}$ \\
0.25 & $0.428 T^{-0.570}$ \\
0.5 & $0.425 T^{-0.478}$ \\
0.75 & $0.400 T^{-0.383}$ \\
\hline
\end{tabular}

Fig. 5 Log-log plots of the maximum growing wavenumber, with time $T$ for $m=0,1 / 4,1 / 2$, and $3 / 4$. The dashed curves are the large time asymptotic solutions given by Eq. (54)

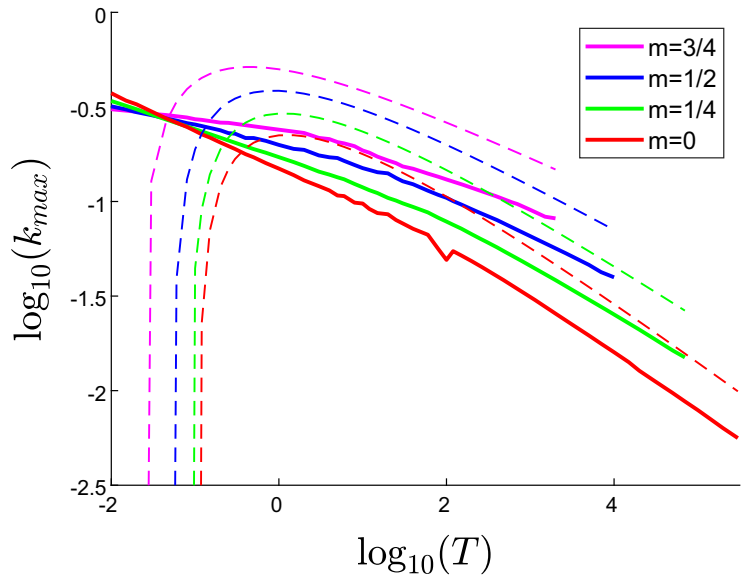

In Table 3, we represent the large time numerically obtained trends for $k_{\max }$ for different values of $m$. Using Table 3, we can obtain the estimates

$k_{\max } \sim T^{0.167 m-0.3}$,

which has similar powers to those predicted by the linear stability theory, namely $T^{(m-2) / 6}$. 
Table 3 Large time numerical trends for $k_{\max }$ for different values of $m$

\begin{tabular}{ll}
\hline$m$ & $k \max$ \\
\hline 0 & $0.251 T^{-0.30}$ \\
0.25 & $0.302 T^{-0.27}$ \\
0.5 & $0.302 T^{-0.22}$ \\
0.75 & $0.302 T^{-0.175}$ \\
\hline
\end{tabular}

\section{Conclusions}

In this study, we have examined the stability of classical diffusive profiles with regard to a Rayleigh-Taylor instability in a two-dimensional vertically oriented porous medium, for a class of initial concentration profiles.

When a species is present in the upper half of the domain such that its far-field concentration vertically tends to $(-X)^{m}$ where $X$ is the vertical coordinate, and absent from the bottom half of the domain, then the base state diffusive concentration profile has been obtained and we found that the eigenfunctions of the perturbations grow like $\exp \left(\omega_{0} T^{(m+1) / 2}+\omega_{1} \ln (T)\right)$ for large times $T$ where $\omega_{0}$ and $\omega_{1}$ are constants. Further, the growth rate defined by $(1 / A)(\mathrm{d} A / \mathrm{d} T)$ decays like $c_{1} T^{(m-1) / 2}+c_{2} T^{(m-2) / 3}$ which was found to be in reasonable agreement with results obtained from a large time numerical linear stability analysis.

The large time linear stability analysis also predicted that the maximum growing wavenumber decays like $T^{(m-2) / 6}$. The large time numerical linear stability analysis showed the same scalings although there was a difference in the magnitudes. The biggest differences occurred near $m=0$ and $m=1$. One notes that the scalings for the growth rates when $m=0$ and $m=1$ are in agreement with the numerical predictions by Cowell et al. [25]. These analytical results should help to provide understanding for the stability of more complex density profiles.

Acknowledgements The authors would like to thank the editor and the referees for their comments which have improved this paper.

\section{Appendix A: Useful limits}

For large $s$, we have the series solutions

$\Omega_{1}(s)=\frac{\Gamma(m+1)}{2^{m+1} \Gamma\left(1+\frac{m}{2}\right)} \sum_{n=0}^{\infty} \frac{s^{m-2 n}}{n ! \Gamma(m+1-2 n)}$

and

$\Omega_{2}(s)=\frac{2^{m+1} \mathrm{e}^{-s^{2} / 4}}{\sqrt{\pi} \Gamma(m+1)} \sum_{n=0}^{\infty} \frac{(-1)^{n} \Gamma(2 n+m+1)}{n ! s^{2 n+m+1}}$

where $\Gamma$ is the gamma function, see Eq. (6.1.1) in Abramowitz and Stegun [31]. For small $s$, we have the series solutions

$\Omega_{1}(s)=\frac{\Gamma\left(\frac{m+1}{2}\right)}{2 \sqrt{\pi}} \sum_{n=0}^{\infty} \frac{s^{2 n+1}}{(2 n+1) ! \Gamma\left(\frac{m+1}{2}-n\right)} \quad$ and $\quad \Omega_{2}(s)=\sum_{n=0}^{\infty} \frac{(-s)^{n}}{n ! \Gamma\left(1+\frac{m-n}{2}\right)}$.

\section{References}

1. Rayleigh L (1883) Investigation of the character of the equilibrium of an incompressible heavy fluid of variable density. Proc Lond Math Soc 14:170-177 
2. Taylor GI (1950) The instability of liquid surfaces when accelerated in a direction perpendicular to their planes. Proc R Soc Lond A 201:192-196

3. Sharp DH (1984) An overview of Rayleigh-Taylor instability. Physica D 12:3-18

4. Boffetta G, Mazzino A (2017) Incompressible Rayleigh-Taylor turbulence. Ann Rev Fluid Mech 49:119-143

5. Conrad CP, Molnar P (1997) The growth of Rayleigh-Taylor-type instabilities in the lithosphere for various rheological and density structures. Geophy. J. Int. 129:95-112

6. Gerya TV, Yuen DA (2003) Rayleigh-Taylor instabilities from hydration and melting propel 'cold plumes' at subduction zones. Earth Planet Sci Lett 212:47-62

7. Huang CS, Kelley MC, Hysell DL (1993) Nonlinear Rayleigh-Taylor instabilities, atmospheric gravity waves and equatorial spread F. J Geophys Res 98:15631-15642

8. De Wit A (2016) Chemohydrodynamic patterns in porous media. Philos Trans R Soc A 374:20150419

9. Cabot WH, Cook AW (2006) Reynolds number effects on Rayleigh-Taylor instability with possible implications for type-Ia supernovae. Nat Phys 2:562-568

10. Horton CW, Rogers FT Jr (1945) Convection currents in porous media. J Appl Phys 20:367-369

11. Lapwood ER (1948) Convection of a fluid in a porous medium. Proc Camb Philos Soc 44:508-521

12. De Wit A (2001) Fingering of chemical fronts in porous media. Phys Rev Lett 87:054502

13. Trevelyan PMJ, Almarcha C, De Wit A (2011) Buoyancy-driven instabilities of miscible two-layer stratifications in porous media and Hele-Shaw cells. J Fluid Mech 670:38-65

14. Martin J, Rakotomalala N, Salin D (2002) Gravitational instability of miscible fluids in a Hele-Shaw cell. Phys Fluids 14:902-905

15. Menand T, Woods A (2005) Dispersion, scale, and time dependence of mixing zones under gravitationally stable and unstable displacements in porous media. Water Resour Res 41:W05014

16. Hill S (1952) Channelling in packed columns. Chem Eng Sci 1:247-253

17. Wooding RA (1969) Growth of fingers at an unstable diffusing interface in a porous medium or Hele-Shaw cell. J Fluid Mech 39:477-495

18. Fernandez J, Kurowski P, Petitjeans P, Meiburg E (2002) Density-driven unstable flows of miscible fluids in a Hele-Shaw cell. J Fluid Mech 451:239-260

19. Lawrie AGW, Dalziel SB (2011) Turbulent diffusion in tall tubes. II. Confinement by stratification. Phys Fluids 23:085110

20. Davies Wykes MS, Dalziel SB (2014) Efficient mixing in stratified flows: experimental study of Rayleigh-Taylor unstable interfaces within an otherwise stable stratification. J Fluid Mech 756:1027-1057

21. Biferale L, Boffetta G, Mailybaev AA, Scagliarini A (2018) Rayleigh-Taylor turbulence with singular nonuniform initial conditions. Phys Rev Fluids 3:092601(R)

22. Gopalakrishnan SS, Carballido-Landeira J, Knaepen B, De Wit A (2018) Control of Rayleigh-Taylor instability onset time and convective velocity by differential diffusion effects. Phys Rev E 98:011101(R)

23. Lemaigre L, Budroni MA, Riolfo LA, Grosfils P, De Wit A (2013) Asymmetric Rayleigh-Taylor and double-diffusive fingers in reactive systems. Phys Fluids 25:014103

24. De Wit A (2020) Chemohydrodynamic patterns and instabilities. Ann Rev Fluid Mech 52:531-555

25. Cowell S, Kent J, Trevelyan PMJ (2020) Rayleigh-Taylor instabilities in miscible fluids with initially piecewise linear density profiles. J Eng Math 121:57-83

26. Tan CT, Homsy GM (1986) Stability of miscible displacements in porous media: rectilinear flow. Phys Fluids 29:3549-3556

27. Gandhi J, Trevelyan PMJ (2014) Onset conditions for a Rayleigh-Taylor instability with step function density profiles. J Eng Math $86: 31-48$

28. Kim MC (2015) Linear stability analysis on the onset of the Rayleigh-Taylor instability of a miscible slice in a porous medium. $\mathrm{J}$ Eng Math 90:105-118

29. Ben Y, Demekhin EA, Chang HC (2002) A spectral theory for small amplitude miscible fingering. Phys Fluids 14:999-1010

30. Nield DA, Bejan A (2006) Convection in porous media. Springer, New York

31. Abramowitz A, Stegun IA (1972) Handbook of mathematical functions. Dover, New York

32. Fulton SR, Ciesielski PE, Schubert WH (1986) Multigrid methods for elliptic problems: a review. Mon Weather Rev 114:943-959

33. Lin S-J, Rood RB (1996) Multidimensional flux-form semi-Lagrangian transport schemes. Mon Weather Rev 124:2046-2070

34. Holdaway D, Kent J (2015) Assessing the tangent linear behaviour of common tracer transport schemes and their use in a linearised atmospheric general circulation model. Tellus A 67:27895

Publisher's Note Springer Nature remains neutral with regard to jurisdictional claims in published maps and institutional affiliations. 